\section{Comparison between Lipofectamine RNAiMAX and GenMute transfection agents in two cellular models of human hepatoma}

\author{
Clarissa Berardo, Veronica Siciliano, \\ Laura G. Di Pasqua, Plinio Richelmi, \\ Mariapia Vairetti, Andrea Ferrigno \\ Department of Internal Medicine and \\ Therapeutics, University of Pavia, Italy
}

\begin{abstract}
RNA interference is a powerful approach to understand gene function both for therapeutic and experimental purposes. Since the lack of knowledge in the gene silencing of various hepatic cell lines, this work was aimed to compare two transfection agents, the liposome-based Lipofectamine ${ }^{T M}$ RNAiMAX and the HepG2-specific, polymer-based GenMute ${ }^{\mathrm{TM}}$, in two cellular models of human hepatoma, HepG2 and Huh7.5. In the first part, we assessed transfection efficiency of a fluorescent Cy3-labeled negative control siRNA by cell imaging analysis; we found that cells treated with GenMute present a higher uptake of the fluorescent negative control siRNA when compared to Lipofectamine RNAiMAX-trasfected cells, both in HepG2 and in Huh7.5 cells. In the second part, we evaluated GAPDH silencing with the two transfection reagents by RT-PCR finding similar GAPDH mRNA expression after each transfection treatment. Finally, we measured cell viability by the MTT assay, observing that cells transfected with GenMute have a higher viability with respect to Lipofectamine RNAiMAXadministered cells. These results suggest that GenMute reagent might be considered the most suitable transfection agent for hepatic gene silencing.
\end{abstract}

\section{Introduction}

Gene silencing mediated by RNA interference (RNAi) is a broadly used approach for both molecular studies and therapy. ${ }^{1}$ RNAi is a biological process mediated by double-stranded RNA, ${ }^{2}$ referred as small non-coding RNAs (20-30 nucleotides), such as short interfering RNAs (siRNAs). ${ }^{3}$ The administered siRNAs face several difficulties in reaching their target: they have to pass cellular membranes, ${ }^{4}$ and to avoid enzymatic $^{5}$ or immune-mediated ${ }^{6}$ degrada- tion. To overcome these obstacles, the use of nanoparticles is considered the best choice to deliver siRNAs. According to the material used, nanoparticles are subdivided in inorganic, organic, viral or hybrid nanoparticles. Among the organic nanoparticles, there are micelles, liposome, proteinbased carries, dendrimers, polymers and cyclodextrins. $^{7}$

The delivery systems considered in this study are the liposome-based Lipofectamine $^{\mathrm{TM}}$ RNAiMAX from Thermofisher Scientific (Waltham, MA, USA) and GenMute ${ }^{\mathrm{TM}}$ from SignaGen (Gaithersburg, MD, USA). The lipofectamine system is based on cationic lipid formulation and it is one of the most used transfection reagents, because of its efficacy on a wide range of cell types. The basic structure of cationic lipids consists of a phospholipid bilayer, with a positively charged head group and one or two hydrocarbon tails. The inner polar head creates electrostatic interactions with the phosphate backbone of the nucleic acid. The positive surface charge of the liposomes allows their endocytosis through the negatively charged cell membrane. According to the chemical structure of the head and tail groups, the efficiency and the toxicity of the liposomes can vary: weakly hyprophilic head and long alkyl chains improved transfection efficiency, but are more toxic than neutral liposomes. ${ }^{8,9}$ On the contrary, GenMute is a novel non-liposomal delivery system; GenMute is sold in different versions optimized for various cell lines including hepatoma. It consists in a biodegradable polymer chemically adapted with defined hydrophobic groups, to induce $\mathrm{pH}$-dependent conformational changes at physiological $\mathrm{pH}$. These arrangements enable a strong stabilization of siRNA complex and guarantee high-rate intracellular delivery.

Considering that there is not much literature on the comparison of RNAi transfection technologies in hepatoma cells, this study had two goals: the first one was to compare two different delivery systems in two hepatoma cell lines of human origin (HepG2 and Huh7.5) by means of a fluorescent dye uptake methodology; the second one was to setup GAPDH gene silencing in the same cell cultures using both transfecting agents.

\section{Materials and Methods}

\section{Cell culture}

HepG2 cells were grown in Minimum Essential Medium EAGLE plus 10\% Fetal Bovine Serum (FBS), 1\% glutamine, 1\%
Correspondence: Laura Giuseppina Di Pasqua, Department of Internal Medicine and Therapeutics, University of Pavia, via A. Ferrata 9/A, 27100 Pavia, Italy.

Tel. +39.0382.986451

E-mail: lauragiuseppin.dipasqua01@universitadipavia.it

Key words: siRNA; Lipofectamine RNAiMAX; GenMute; HepG2; Huh7.5.

Contributions: $\mathrm{CB}$, drafting, data acquisition, data analysis, final approval; VS, LGDP, manuscript revision, data acquisition and analysis, final approval; PR, manuscript revision, data interpretation, final approval; $\mathrm{MV}, \mathrm{AF}$, conception and design, data interpretation, final approval. CB and VS contributed equally.

Conflict of interest: all authors declare no conflict of interest related to this publication.

Acknowledgements: We thank Ms. Micaela Ascoli and Ms. Nicoletta Breda for their skilful technical assistance.

Received for publication: 30 May 2019. Accepted for publication: 18 July 2019.

This work is licensed under a Creative Commons Attribution-NonCommercial 4.0 International License (CC BY-NC 4.0).

(C) Copyright: the Author(s), 2019

Licensee PAGEPress, Italy

European Journal of Histochemistry 2019; 63:3048 doi:10.4081/ejh.2019.3048

sodium pyruvate and $1 \%$ antibiotic-antimycotic solution (penicillin, streptomycin and amphotericin B).

Huh7.5 cells were grown in Dulbecco's Modified Eagle Medium (DMEM) supplemented with 10\% FBS, 1\% Non Essential Amino Acids and 1\% antibiotic-antimycotic solution (penicillin, streptomycin and amphotericin B). All cells were cultured at $37^{\circ} \mathrm{C}$ and $5 \% \mathrm{CO}_{2}$.

\section{Cy3-labeled siRNA uptake}

HepG2 and Huh7.5 cells were cultured in 96-well plate at 50,000 cells/well for $24 \mathrm{~h}$ in their culture medium prior to the experiment. On the day of transfection, cells were washed once with sterile phosphatebuffered saline (PBS) and the culture medium was added with the transfection reagents (Lipofectamine ${ }^{\mathrm{TM}}$ RNAiMAX, Cat\#: 13778-100; or GenMute ${ }^{\mathrm{TM}}$, Cat\#: SL100568-HepG2) plus 5, 10 or $20 \mathrm{nM}$ of the Silencer ${ }^{\circledR}$ Cy3-labeled negative control siRNA (Cy3-siRNA) (Cat\#: AM4621).

For Lipofectamine RNAiMAX, the culture medium was replaced by $100 \mu \mathrm{l}$ of Opti-MEM (1X) plus GlutaMAX and 5\% fetal bovine serum just before the Lipofectamine RNAiMAX reagent addi- 
tion. Both the reagent and the Cy3-siRNA at different concentrations were diluted $1: 1$ in serum-free Opti-MEM and incubated for 5 $\mathrm{min}$ at room temperature, prior to be added to each well.

For GenMute treatment, cells were incubated in $100 \mu \mathrm{L}$ of fresh culture medium $30 \mathrm{~min}$ before the transfection. For GenMute reagent preparation, once the transfection buffer has been diluted, it was incubated with the different concentrations of Cy3-siRNA for $15 \mathrm{~min}$ at room temperature and, then, added to each well. After 5 and $24 \mathrm{~h}$ of incubation at $37^{\circ} \mathrm{C}$ and $5 \% \mathrm{CO}_{2}$, cells were washed once with PBS. Nuclei were incubated for $15 \mathrm{~min}$ with $1 \mu \mathrm{g} / \mu \mathrm{L}$ of the fluorescent DNA probe Hoechst 33342 (Thermofisher Scientific). Then, cells were washed once with PBS and observed by means of the fluorescent cell imager ZOE (Bio-Rad Laboratories, Hercules, CA, USA). Images were analyzed by ImageJ software, using the subtraction background method.

\section{GAPDH silencing}

HepG2 and Huh7.5 cells were seeded in 6-well plate at 2.5-3 $\times 10^{5}$ cells/well for 24 $\mathrm{h}$ in their respective culture medium before the experiments. The silencer Select GAPDH siRNA (Cat\#:4390843) and the Silencer Select Negative Control \#1 siRNA (Cat\#: 4390843) (both at $10 \mathrm{nM}$ ) were transfected by using Lipofectamine RNAiMAX Transfection Reagent or GenMute for HepG2, as previously described.

\section{RT-PCR}

After 24 and $48 \mathrm{~h}$, total RNA was isolated from HepG2 and Huh7.5 cells with TRI reagent (Sigma-Aldrich, St. Louis, MO, USA), following the Chomczynski method. ${ }^{10}$ RNA was quantified by measuring the absorbance at $260 / 280 \mathrm{~nm}$ with T92+ UV Spectrophotometer. ${ }^{11}$ The cDNA was generated using iScript Supermix (BioRad). ${ }^{12}$

The qPCR reactions were performed by CFX96TM Real-Time System (Bio-Rad) using $5 \mu \mathrm{L}$ of SsoAdvancedTM SYBR ${ }^{\circledR}$ Green Supermix (Bio-Rad), $0.5 \mu \mathrm{L}$ of each oligonucleotide primer $(10 \mathrm{pmol} / \mu \mathrm{L})$ and 1 $\mu \mathrm{L}$ of cDNA $(2.5 \mathrm{ng} / \mu \mathrm{L})$ to reach a final volume of $10 \mu \mathrm{L} /$ well.

GAPDH, USP28, TUBA1A and RPS9 gene amplification efficiencies were established by means of calibration curves (100.1\%, 103.8\%, 181.5\%, 110.8\%, respectively). The expression of the reference genes remained constant in the considered experimental groups. The amplicon context sequence of the primers (Bio-Rad) are reported in Table 1 . The results were normalized to the endogenous controls, and fold change of the gene expression was calculated using threshold cycle $(\mathrm{Ct})$ values.

\section{Cell viability}

Viability of HepG2 and Huh7.5 cells transfected with GAPDH siRNA or nega- tive control siRNA by either Lipofectamine RNAiMAX or GenMute as previously described was assessed by 3-(4,5dimethylthiazol-2-yl)-2,5-diphenyltetrazolium bromide (MTT) assay. Cells were washed once with PBS and $100 \mu \mathrm{L}$ of serum-free medium containing MTT 1.2 $\mathrm{mM}$ was added to each well. For negative control, $4 \mu \mathrm{L}$ of Tryton X $25 \%$ were added to three selected wells prior to the treatment with MTT. After $2 \mathrm{~h}$ of incubation at $37^{\circ} \mathrm{C}$ at $5 \% \mathrm{CO}_{2}$, formazan crystals were dissolved in $100 \mu \mathrm{L}$ of DMSO. The absorbance was measured at $540 \mathrm{~nm}$ by means of a microplate spectrophotometer. ${ }^{13}$

\section{Statistical analysis}

Statistical analysis was performed by means of R Statistical software (ver. 3.5.2) and the graphical interface R Studio (ver. 1.0.143). Parametric data were analyzed by
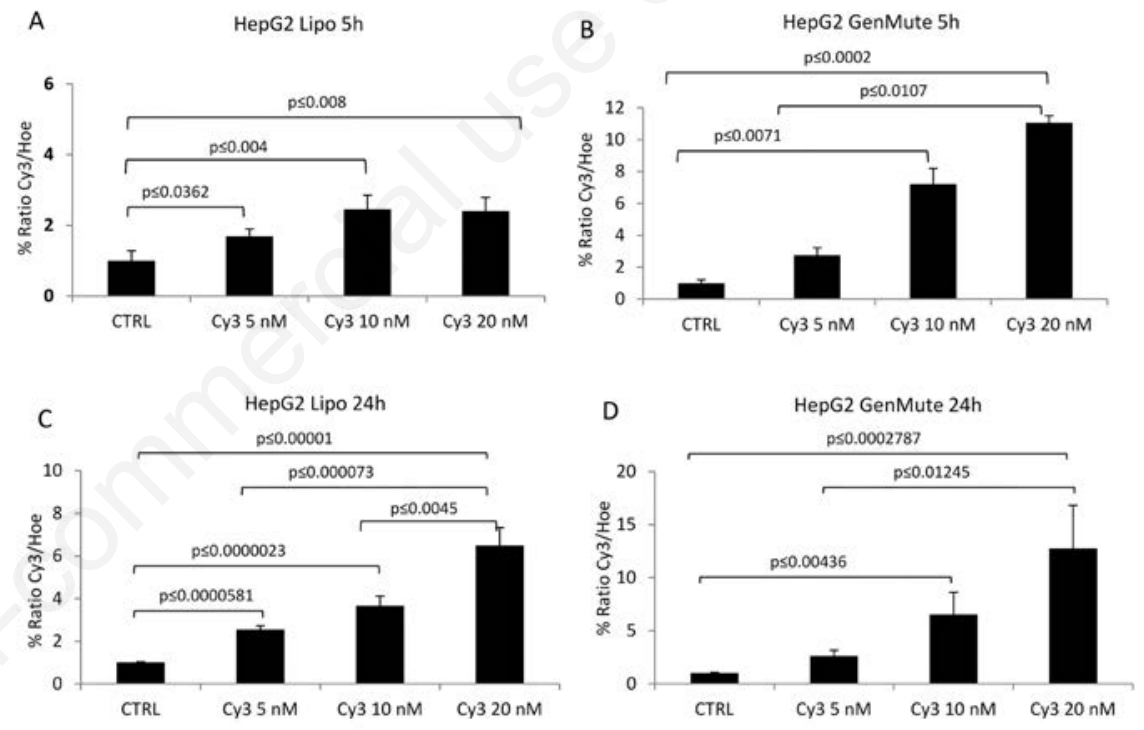

Figure 1. Cy3-labeled siRNA uptake by HepG2 cells after treatment with transfecting agents for 5 or $24 \mathrm{~h}$. A) Lipofectamine RNAiMAX treatment for $5 \mathrm{~h}$; B) GenMute treatment for $5 \mathrm{~h}$; C) Lipofectamine RNAiMAX treatment for $24 \mathrm{~h}$; D) GenMute treatment for $24 \mathrm{~h}$. Values are expressed as arithmetic mean $\pm \mathrm{SE}$.

Table 1. Amplicon sequences of primers used in this study. Glyceraldehyde-3-Phosphate Dehydrogenase (GAPDH) was the gene of interest, while Ubiquitin Specific Peptidase 28 (USP28), Tubulin alpha 1A (TUBA1A) and Ribosomal Protein Subunit 9 (RPS9) were used as the reference genes.

\begin{tabular}{|c|c|}
\hline Gene & Amplicon sequence \\
\hline GAPDH & $\begin{array}{l}\text { GTATGACAACGAATTTGGCTACAGCAACAGGGTGGTGGACCTCATGGCCCACATGGCCTCCAAGGAGTAAGACCCCTGGACCACCAGCCCCAGCAAGAGCACA } \\
\text { AGAGGAAGAGAGAGACCCTCACTGCTGGGGAGTCCCTGCCACA }\end{array}$ \\
\hline USP28 & AACATCACCCTCCACCATGGCCCCTTCCAAACACTCGTCTAAGTTGCGATAACCGTTTACCTGAAGAGGATACTGGCCGAAGGTCTCATTGTTACAAAAGGGTT \\
\hline TUBA1A & $\begin{array}{l}\text { GCGAATTCGGTCCAACACGAGGTCAATGATCTCCTTGCCAATGGTGTAGTGCCCTCGGGCATAGTTATTGGCAGCATCTTCTTTGCCTGTGATAAGTTGCTCAGGGT } \\
\text { GGAAGAGCTGGC }\end{array}$ \\
\hline RPS9 & $\begin{array}{l}\text { CCGCCCGGGAACTGCTGACGCTTGATGAGAAGGACCCACGGCGTCTGTTCGAAGGCAACGCCCTGCTGCGGCGGCTGGTCCGCATTGGGGTGCTGGATGAGGG } \\
\text { CAAGATGAAGCTGGATTACATCCTGGGCCTGA }\end{array}$ \\
\hline
\end{tabular}


Student $t$-test or Tukey's HSD Test. Nonparametric data were analyzed by Welch's test, Wilcoxon's Test or Dunn's Test.

\section{Results}

\section{Cy3-labeled negative control siRNA transfection}

In both cell lines, a concentrationdependent uptake in Cy3-labeled negative control siRNA uptake occurred (Figure 1). After $5 \mathrm{~h}$ of incubation, using Lipofectamine RNAiMAX in HepG2 cells, the ratio $\mathrm{Cy} 3 / \mathrm{Hoe}$ was significantly higher between the Cy3-siRNA treated cells and the control (Figure 1A), and the significance increased after $24 \mathrm{~h}$ (Figure 1C). We assisted to a significant increase in Cy3siRNA uptake also using GenMute, at 5 (Figure 1B) and especially at $24 \mathrm{~h}$ of treatment respect with controls (Figure 1D). Comparing the data obtained from the transfection with the two reagents, we observed that the $\mathrm{Cy} 3 / \mathrm{Hoe}$ ratio was more consistent using GenMute rather than Lipofectamine RNAiMAX (Figure 2). In fact, the GenMute-mediated siRNA uptake was significantly higher exclusively in the 5-h treatment with Cy3-siRNA at both 10 and $20 \mathrm{nM}(\mathrm{P} \leq 0.005$ and $\mathrm{P} \leq 0.0105$, respectively); at $24 \mathrm{~h}$ the fluorescent signal of GenMute was 2-fold higher at $10 \mathrm{nM}$ and at $20 \mathrm{nM}$ when compared to the respective concentrations for Lipofectamine RNAiMAX, although the difference was not significant (Figure 2).

In Huh7.5 cells, Lipofectamine RNAiMAX transfection was significant at 5 h only for $20 \mathrm{nM} \mathrm{Cy3-siRNA} \mathrm{(Figure} \mathrm{3A),}$ while at $24 \mathrm{~h}$ became significant also at 5 and $10 \mathrm{nM}$ (Figure 3C). The transfection mediated by GenMute was significantly higher for all Cy3-siRNA concentrations at both $5 \mathrm{~h}$ (Figure 3B) and $24 \mathrm{~h}$ (Figure 3D) with respect to the relative controls, while a timedependent increase in the transfection levels increased. Comparing the data of the two transfectants, we found that in cells treated with GenMute the $\mathrm{Cy} 3 /$ Hoe ratio was more consistent than in cells treated with Lipofectamine RNAiMAX. In fact, at all Cy3-siRNA concentrations the uptake of fluorescent probe was significantly higher using GenMute transfection when compared to Lipofectamine RNAiMAX (Figure 4).

\section{GAPDH silencing}

In HepG2 cells, GAPDH silencing obtained by using GenMute was significant with respect to its relative control only after $24 \mathrm{~h}$ of treatment with the transfection agent (Figure 5A). On the contrary, GAPDH was significantly silenced with Lipofectamine
RNAiMAX after $48 \mathrm{~h}$ of siRNAs administration (Figure 5B). Comparing the data obtained by the two reagents, we found that, at $48 \mathrm{~h}$, the silencing mediated by Lipofectamine RNAiMAX was significantly higher respect to that with GenMute (Figure 6). In Huh7.5 cells, GAPDH mRNA expression was reduced after 24 and
$48 \mathrm{~h}$ with both transfectants (Figure 7). Differently from what observed in HepG2 silencing, no significant differences were found comparing the two reagents in Huh7.5 cells (Figure 8).

\section{Cell viability}

After $24 \mathrm{~h}$ of either GAPDH or negative

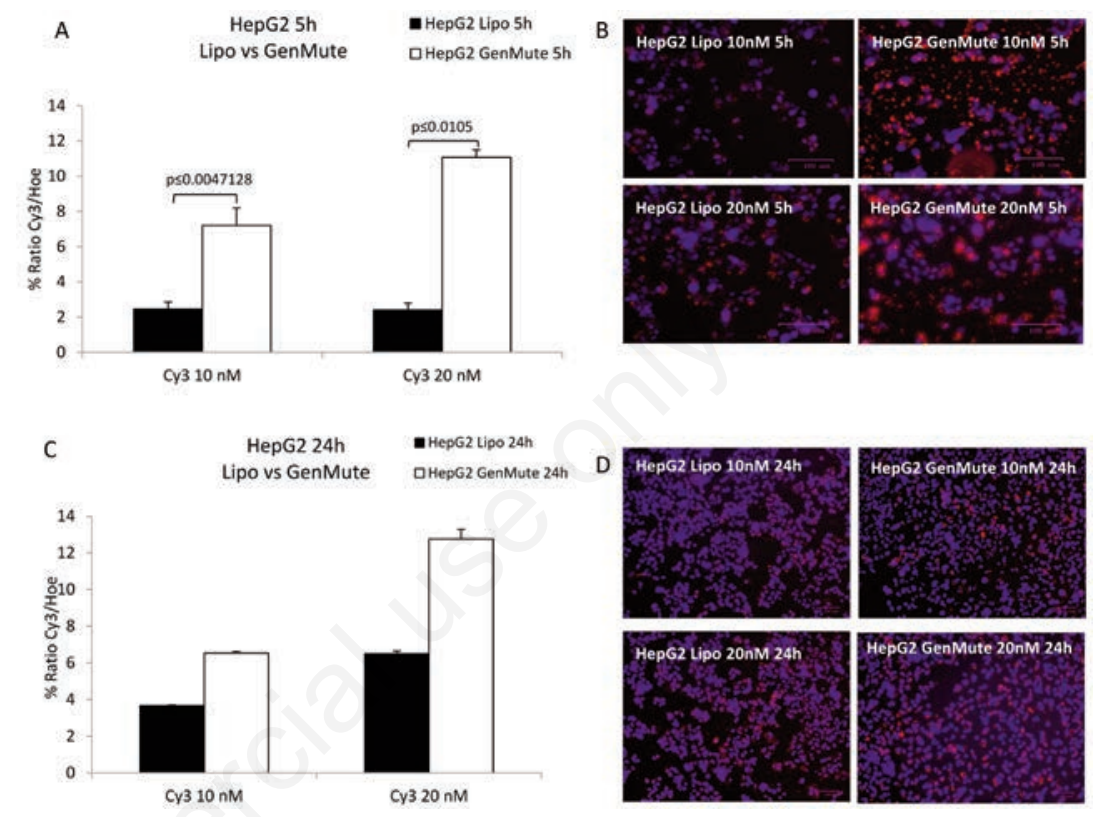

Figure 2. Direct comparison among $\mathrm{Cy} 3$ uptake in HepG2 cells treated with the transfecting agents Lipofectamine RNAiMAX and GenMute. Graphical representation after 5 (A) and $24 \mathrm{~h}(\mathrm{C})$ of incubation and respective cell imaging (B-D). Values are expressed as arithmetic mean $\pm \mathrm{SE}$.
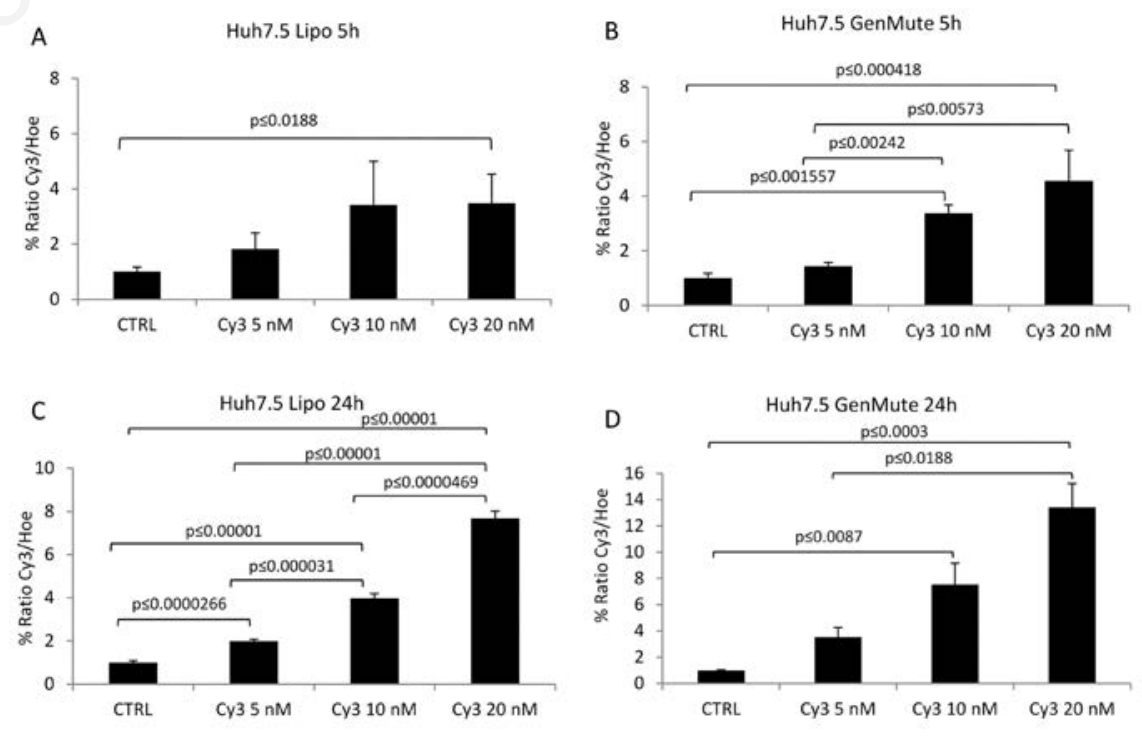

Figure 3. Cy3-labeled siRNA uptake by Huh7.5 cells after treatment with transfecting agents for 5 or $24 \mathrm{~h}$. A) Lipofectamine RNAiMAX treatment for $5 \mathrm{~h}$; B) GenMute treatment for $5 \mathrm{~h}$; C) Lipofectamine RNAiMAX treatment for $24 \mathrm{~h}$; D) GenMute treatment for $24 \mathrm{~h}$. Values are expressed as arithmetic mean $\pm \mathrm{SE}$. 
control siRNA incubation, cell viability in HepG2 cells was significantly lower in Lipofectamine RNAiMAX treated cells as compared to both GenMute transfected or control cells seeded with EAGLE medium (Figure 9A). After $48 \mathrm{~h}$, the differences among groups were smoothed, even though the negative control siRNA transfected by GenMute displayed a significantly lower viability respect to negative control siRNA transfected by Lipofectamine RNAiMAX and the control cells grown in EAGLE medium (Figure 9B). In Huh7.5 cells, viability was significantly reduced by Lipofectamine RNAiMAX transfection when compared to GenMute or control cells seeded with DMEM medium after $24 \mathrm{~h}$ (Figure 9C) and $48 \mathrm{~h}$ (Figure 9D).

\section{Discussion}

In our first experiments, we compared the transfection efficiency in two immortalized hepatoma cell lines, HepG2 and Huh7.5 cells, by means of two transfection reagents, Lipofectamine RNAiMAX and GenMute. We observed that in both cell lines Cy3-siRNA uptake was significantly greater when using GenMute treatment with respect to Lipofectamine RNAiMAX. Several studies demonstrated that the presence of lateral chains on the vector correlates with a major transfection efficiency, since much more interactions between siRNA molecules and the vector can occur. For this reason, the possibility that siRNA is "unloaded" from the nanoparticle and, consequently, more susceptible to nuclease degradation is reduced. ${ }^{14}$ Moreover, the changes in carrier lateral groups represent a good strategy for the formulation of compounds released to specific cell targets..$^{15-17}$ However, siRNA uptake mediated by Lipofectamine RNAiMAX was greater in Huh7.5 cells than in HepG2 cells. These data confirm the results recently published by Wang et al. according to which the transfection with Lipofectamine RNAiMAX in Huh7.5 was superior than in HepG2 cells (46.12\% versus $24.32 \%$, respectively). As to GenMute, our data confirm that the transfection is more efficient for HepG2 cells, ${ }^{19}$ for which the reagent is specifically designed.

To assess the extent of mRNA silencing mediated by the two transfection reagents, we used the endogenous model gene GAPDH. A decrease in GAPDH mRNA expression was found in hepatoma cell lines with both transfectants when treated with GAPDH siRNA as compared to the nonsilenced control. Except for HepG2 cells after $48 \mathrm{~h}$ of transfection, we did not
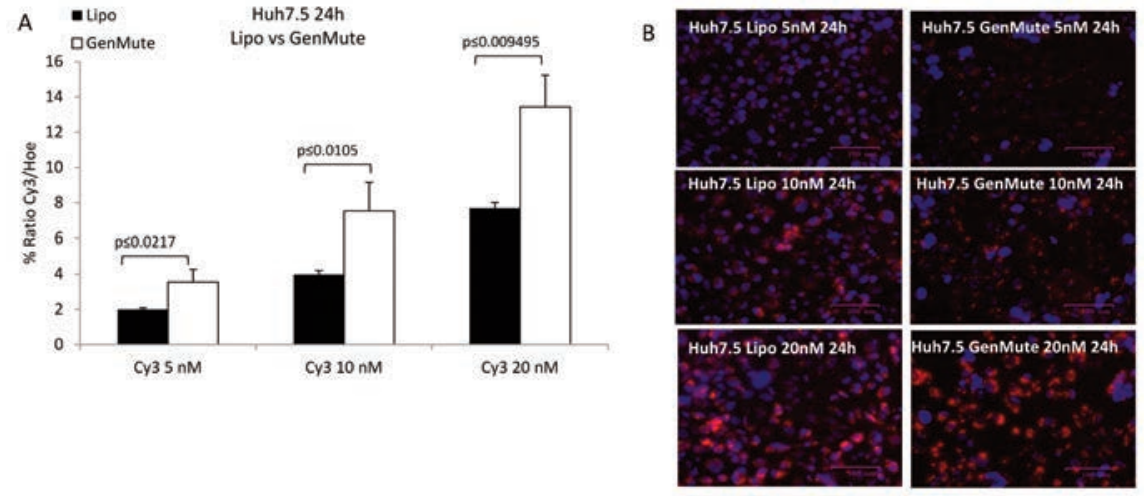

Figure 4. Direct comparison among $\mathrm{Cy} 3$ uptake in Huh7.5 cells treated with the transfecting agents Lipofectamine RNAiMAX and GenMute. Graphical representation (A) and cell imaging $(B)$. Values are expressed as arithmetic mean \pm SE.

observe any appreciable differences when comparing Lipofectamine RNAiMAX and GenMute silencing efficiency. For this comparison, we used a mild concentration of GAPDH siRNA to avoid any effects on cellular viability. In fact, GAPDH is considered a potential therapeutic target since its suppression by antagonists and inhibitors is associated to cell proliferation arrest. ${ }^{19,20}$ Since we had found that GenMute transfected Cy3-siRNA with a significantly higher efficiency, we expected to find a difference with regards to silencing. On the contrary, a correlation between $\mathrm{Cy} 3$ transfection efficiency and GAPDH silencing was not found. In our opinion, the concentration of GAPDH siRNA used in GAPDH silencing experiments $(10 \mathrm{nM})$ was higher enough to deliver saturating amounts of siRNA with both the transfecting agents; consequently, no difference was found in GAPDH silencing. This "saturating effect" was not visible for Cy3 delivery because the fluorescence signal is linear in a wide range of intensity.

After evaluating the transfectant cytotoxicity by MTT assay, we found that cell viability was greater in GenMute treated cells as compared to Lipofectamine RNAiMAX at $24 \mathrm{~h}$. It has been recently shown a correlation between transfection efficiency and cell viability: the higher is the transfection, the higher is the cytotoxicity. According to Neuhaus and collaborators, it occurs independently to the transfectant used, ${ }^{21}$ while Wang et al. observe that it depends on cell type. ${ }^{22}$ Although the presence of lateral groups is associated to greater transfection efficiency, it appears to be harmful for cell viability. ${ }^{14}$ In our work, however, we did not find this correlation. Moreover, following manufacturers' instructions, GenMute-mediated transfection is performed in the "physiological" /suitable culture medium of the cells, while

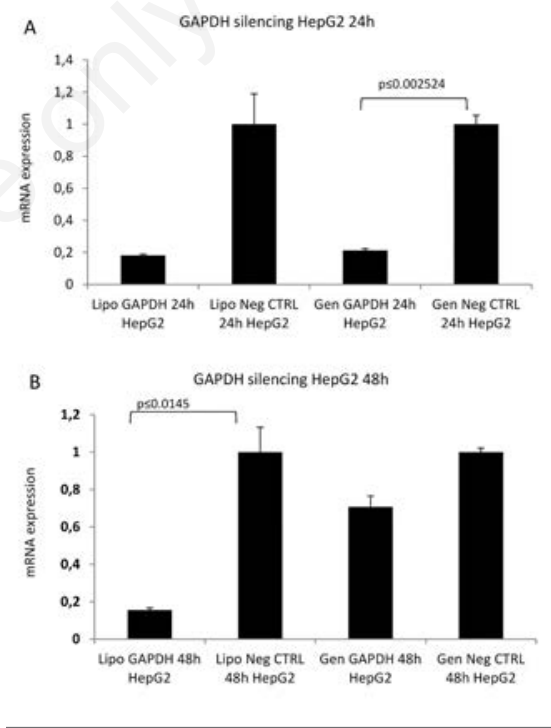

Figure 5. GAPDH silencing mediated by Lipofectamine RNAiMAX and GenMute in HepG2 cells. GAPDH mRNA expression after 24 (A) and $48 \mathrm{~h}(\mathrm{~B})$ of incubation. Values are expressed as arithmetic mean \pm SE.

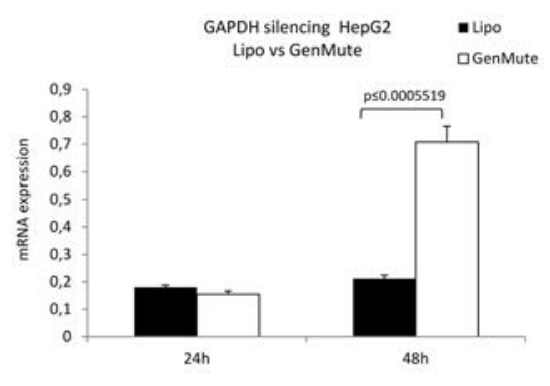
Lipofectamine RNAiMAX and GenMutemediated GAPDH silencing in HepG2 cells after 24 and $48 \mathrm{~h}$ of transfection. Values are expressed as arithmetic mean \pm SE.
Figure 6. Comparison between 
Lipofectamine RNAiMAX works in a nonphysiological medium, Opti-MEM plus GlutaMAX, which requires a reduced serum percentage.

In summary, i) GenMute-mediated Cy3-siRNA uptake was higher than Lipofectamine RNAiMAX; ii) GenMuteGAPDH silencing is comparable to that observe with the "gold standard"
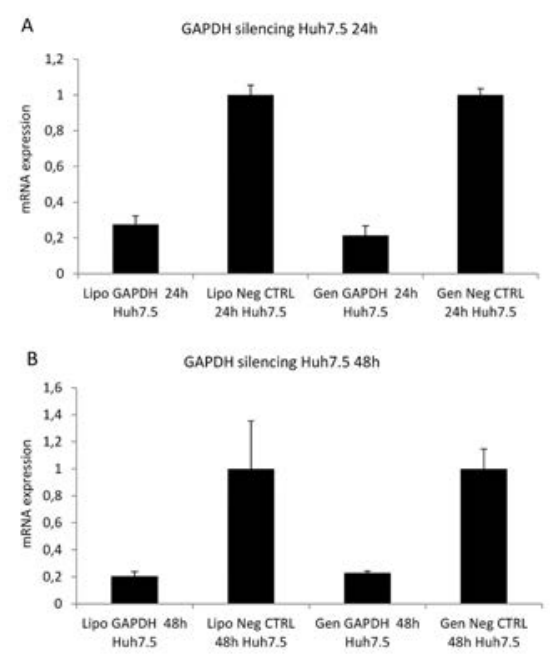

Figure 7. GAPDH silencing mediated by Lipofectamine RNAiMAX and GenMute in Huh7.5 cells. GAPDH mRNA expression after 24 (A) and 48 h (B) of incubation. Values are expressed as arithmetic mean \pm SE.

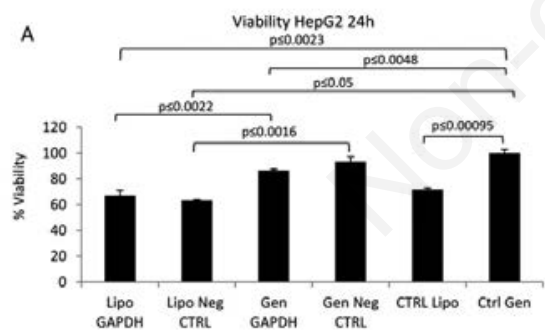

B
Lipofectamine RNAiMAX; and iii) viability in GenMute treated cells was greater than in Lipofectamine RNAiMAX transfected cells. Moreover, GenMute is cheaper than Lipofectamine RNAiMAX: not only for the transfection reagent itself, but also for the specific medium required by Lipofectamine RNAiMAX transfection.

In conclusion, in hepatocellular culture, we support the use of GenMute in comparison with Lipofectamine RNAiMAX to obtain a good viability and a satisfactory uptake of transfection agent during gene silencing.

\section{References}

1. Leung RKM, Whittaker PA. RNA inter-

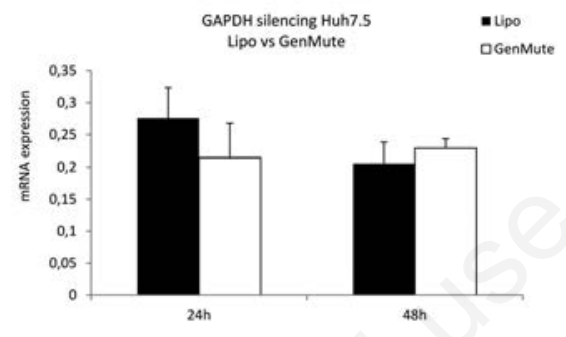

Figure 8. Comparison between Lipofectamine RNAiMAX and GenMutemediated GAPDH silencing in Huh7.5 cells after 24 (A) and $48 \mathrm{~h}$ (B) of transfection. Values are expressed as arithmetic mean \pm SE.
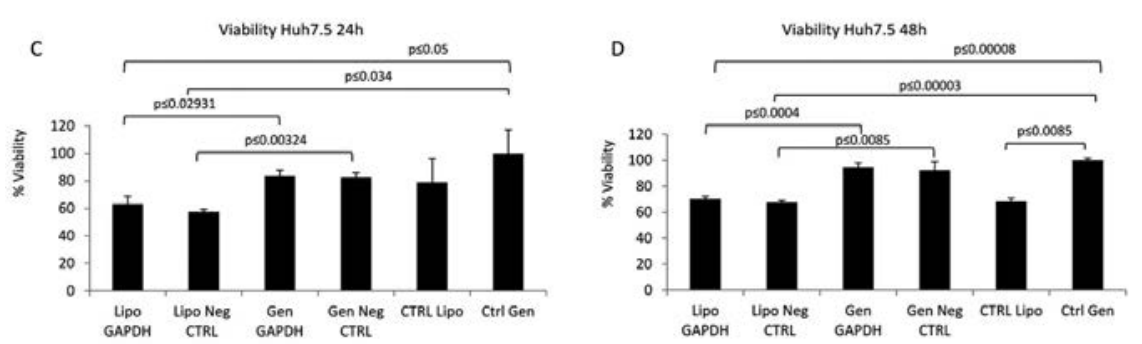

Figure 9. Cell viability in Lipofectamine RNAiMAX and GenMute-treated cells measured by MTT assay. HepG2 cell viability after 24 (A) and $48 \mathrm{~h}$ of incubation (B). Huh7.5 cell viability at $24(\mathrm{C})$ and $48 \mathrm{~h}(\mathrm{D})$ after transfection. Values are expressed as arithmetic mean \pm SE.

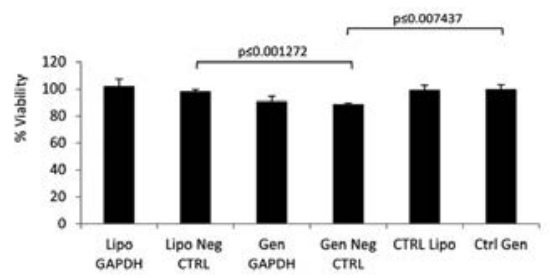
Siciliano V, Rizzo V, Adorini L, et al. The farnesoid $\mathrm{X}$ receptor agonist obeticholic acid upregulates biliary excretion of asymmetric dimethylarginine via MATE-1 during hepatic ischemia/reperfusion injury. PLoS One 2018; 13:e0191430.

13. Riss TL, Moravec RA, Niles AL, Duellman S, Benink HA, Worzella TJ, et al. Cell viability assays. Assay Guidance Manual [Internet]. Bethesda: Eli Lilly \& Company and the National Center for Advancing Translational Sciences; 2004.

14. Wang J, Lu Z, Wientjes MG, Au JL. Delivery of siRNA therapeutics: 
Barriers and carriers. 2010;12:492-503.

15. Semple SC, Akinc A, Chen J, Sandhu AP, Mui BL, Cho CK, et al. Rational design of cationic lipids for siRNA delivery. Nat Biotechnol 2010;28:172-6.

16. Croce ACAC, De Simone U, Vairetti M, Ferrigno A, Boncompagni E, Freitas I, et al. Liver autofluorescence properties in animal model under altered nutritional conditions. Photochem Photobiol Sci 2008;7:1046-53.

17. Hafez IM, Maurer N, Cullis PR. On the mechanism whereby cationic lipids promote intracellular delivery of polynucleic acids. Gene Ther 2001;8:1188-96.
18. SignaGen Laboratoris. Application note: Efficient Gene Silencing in HepG2 Cells Using GenMute ${ }^{\mathrm{TM}}$ siRNA Transfection Reagent. Explorer 2011;1. Available from signagen.com/ Application Notes/Application Note GenM ute_HepG2.pdf

19. Liu S, Sun Y, Jiang M, Li Y, Tian Y, Xue $\mathrm{W}$, et al. Glyceraldehyde-3-phosphate dehydrogenase promotes liver tumorigenesis by modulating phosphoglycerate dehydrogenase. Hepatology 2017;66:631-45.

20. Ganapathy-Kanniappan S. Evolution of GAPDH as a druggable target of tumor glycolysis? Expert Opin Ther Targets 2018;22:295-8.

21. Neuhaus B, Tosun B, Rotan O, Frede A, Westendorf AM, Epple M. Nanoparticles as transfection agents : a comprehensive study with ten different cell lines. RSC Advances 2016;6: 18102-12.

22. Wang T, Larcher LM, Ma L, Veedu RN. Systematic screening of commonly used commercial transfection reagents towards efficient transfection of singlestranded oligonucleotides. Molecules 2018;23:2564. 